\title{
EDITORIAL
}

\section{ACTUACIONES FRENTE A LA DISTRIBUCIÓN INVERSA DE MEDICAMENTOS: LA COOPERACIÓN ES LA CLAVE}

\section{Belén Escribano Romero}

Departamento de Inspección y Control de Medicamentos. Agencia Española de Medicamentos y Productos Sanitarios (AEMPS).

Los medicamentos son bienes de consumo con unas características especiales que los diferencian de otros productos debido al impacto que pueden tener en nuestra salud. Por ello la normativa que los regula incluye unos estrictos controles que se extienden desde el principio de su desarrollo hasta su utilización por los pacientes, con el fin de que reúnan las necesarias garantías.

La Comisión Europea ha celebrado recientemente los 50 años $^{1}$ de la primera normativa europea que reguló las autorizaciones de los medicamentos con el fin de salvaguardar la salud pública, incluyendo las garantías de calidad, seguridad y eficacia.

Los principios de este marco regulador se han mantenido en las distintas etapas de la evolución que se ha producido en este marco normativo durante las últimas décadas, para afrontar nuevos retos, como son los medicamentos falsificados que se abordaron en la Directiva 2011/62/UE ${ }^{2}$ en respuesta a los casos, detectados en varios Estados miembros, de introducción de medicamentos falsificados en el canal legal de suministro y dispensación.

Es preciso considerar además que este marco legal regula un sector, el farmacéutico, de gran importancia económica. En 2013, el comercio de medicamentos en la Unión Europea ascendió a casi $160(156,9)$ billones de euros y las exportaciones casi alcanzaron los $110(107,4)$ billones.

Esta importancia económica del mercado de medicamentos resulta de gran interés, no solo para el desarrollo de operaciones legales sino también para las mafias internacionales que estudian a fondo la normativa vigente buscando posibles huecos en la misma que les permitan desarrollar actuaciones ilegales, tanto de falsificación como de desvío de medicamentos al canal ilegal, en las que se generan importantes ganancias.

En un escenario con las circunstancias del gran mercado único que supone la Unión Europea, favorecido por la aplicación de las nuevas tecnologías, la vía clásica de distribución de medicamentos ha evolucionado hacia cadenas complejas de suministro en las que era difícil asegurar su integridad frente al riesgo de los medicamentos falsificados. Para afrontar la amenaza de la penetración de medicamentos falsificados en el canal legal, como se ha mencionado, se modificó la normativa europea con la Directiva 2001/62/UE para reforzar las garantías de los medicamentos y sus principios activos en su fabricación y, especialmente, en la cadena de distribución.

Los cambios, incluidos en la mencionada directiva, se incorporaron a nuestro ordenamiento jurídico en 2013, y entre ellos es preciso mencionar el concepto de medicamento 
falsificado, que incluye tanto a los que lo son porque su identidad $u$ origen no coincide con lo que declaran en su envase o autorización como a aquellos que lo son porque su historial, relativo a los documentos de su distribución, es falso.

Y es que en los casos detectados e investigados en distintos países de la Unión, se observó, en distintas operaciones ilegales que no solo se introducían en la cadena legal de suministro copias casi idénticas de los medicamentos sino que también se falsificaban los documentos relativos a la cadena de suministro, por lo cual en estos casos, en los que se desconocen las condiciones de conservación y transporte, tampoco es posible asegurar que se hayan mantenido las necesarias garantías de calidad que deben tener los medicamentos.

La experiencia de una comunidad autónoma en casos de tráfico inverso de medicamentos y los riesgos que estas prácticas llevan aparejados para los pacientes se exponen en un artículo de este número ${ }^{3}$. En la distribución inversa, o tráfico inverso, redes organizadas adquieren medicamentos en las oficinas de farmacia para después, de forma ilícita, reintroducirlos a través del canal legal en las entidades de distribución que los exportan, incumpliendo la normativa vigente y ocultando su cadena de distribución previa.

La legislación que regula las oficinas de farmacia en España no contempla entre sus funciones la distribución de medicamentos sino su dispensación, por ello sus obligaciones y funcionamiento son distintos de los que se aplican a los almacenes mayoristas. En algunos países europeos, sin embargo, sí es posible que las farmacias obtengan una autorización adicional como distribuidoras, teniendo en ese caso que cumplir con todas las obligaciones inherentes a las dos actividades.

La cuestión de la posibilidad de desarrollar actividades de distribución por parte de las farmacias en nuestro país fue motivo, hace varios años, de diversas consultas a la Agencia Española de Medicamentos y Productos Sanitarios (AEMPS), que se pronunció al respecto aclarando la ilegalidad del desarrollo de estas prácticas, en una nota de su Dirección dirigida a las comunidades autónomas en febrero de 2011. Posteriormente, en junio de 2012, el Tribunal de Luxemburgo se pronunció de forma similar en el marco de un procedimiento penal que se seguía, frente a un farmacéutico italiano, por una actividad de venta al por mayor de medicamentos que se realizaba sin disponer de la autorización prevista por la legislación italiana ${ }^{4}$.

Como demuestra este caso, la distribución inversa no es un fenómeno aislado que afecte únicamente a España. Las diferencias de precio de los medicamentos, especialmente entre los países del norte y sur de Europa, proporcionan elevados beneficios a las distintas entidades que intervienen en el denominado 'comercio paralelo' y ha provocado no pocos quebraderos de cabeza a las autoridades de los países que sufren importantes problemas de suministro causados por el mismo. Esta actividad es legítima en el gran mercado único europeo, salvo cuando provoca un problema de salud pública en el país de origen, como son los desabastecimientos de medicamentos.

En el artículo 36 del actual Tratado de Funcionamiento de la Unión Europea, se indica la posibilidad de establecer restricciones cuantitativas a la libre circulación de mercancías, recogida en los artículos 34 y 35. Esta limitación es posible cuando su objeto es la protección de la salud y la vida de las personas. Es en este artículo, en el que se ha basado la AEMPS para exigir, desde julio de 2012, la notificación previa ${ }^{6}$ de los envíos intracomunitarios de determinados medicamentos cuya falta de suministro tiene graves consecuencias en los pacientes por la ausencia de alternativas terapéuticas en nuestro mercado. Esta información previa a los envíos a otros Estados miembros hace posible actuar de una forma preventiva, proporcionada y más eficiente, antes de que se produzca la salida de medicamentos que pueda provocar un problema de suministro con repercusión asistencial. Actuaciones similares, siguiendo el ejemplo de España, se han empezado a desarrollar por otros países. 
La actividad de distribución inversa ha sido combatida desde hace varios años por todos los servicios de inspección de las comunidades autónomas así como por la AEMPS, ya que, como se describe en el mencionado artículo ${ }^{3}$, estas prácticas ilegales causan importantes problemas de suministro posibilitando además la entrada de medicamentos falsos, en cuanto a su historial y sin garantías respecto a su conservación y, por lo tanto, a su calidad, en el canal legal de los países a los que se exportan los medicamentos que se desvían mediante estas prácticas.

Por ello, por parte de todas las autoridades competentes, se han realizado todo tipo de actuaciones para evitar el desarrollo de estas prácticas que se pueden englobar en tres tipos:

- Actualización del marco regulador: cabe destacar la introducción, en el año 2011, entre las faltas muy graves de la entonces Ley 29/2006 de garantías de medicamentos y productos sanitarios, ahora Real Decreto Legislativo $1 / 2015$, de 24 de julio, por el que se aprueba el texto refundido de la Ley de garantías y uso racional de los medicamentos y productos sanitarios $^{7}$, en su artículo 111.2 c) de dos faltas: una relacionada con el desarrollo de la actividad de distribución por las oficinas de farmacia y otra relativa a la distribución a otros países de medicamentos con problemas de suministro que tengan repercusión asistencial. Esta calificación de muy grave era necesaria por una parte para que la ilegalidad de estas prácticas quedara tipificada en nuestro marco legal pero también porque las cuantías de las sanciones no eran disuasorias y se reincidía en la comisión de estas prácticas ilegales que reportan pingües beneficios.

También en la última reforma del Código Penal $^{8}$, aprobada este año 2015 y que entró en vigor el pasado 1 de julio, en su artículo 362 , se incluyó el concepto de medicamento falsificado en cuanto a su historial, en línea con la Directiva 2011/62/UE y la normativa nacional de transposición. Esta modificación sin duda también va a ayudar a desincentivar estas prácticas.
Pero además en la transposición de la normativa europea ${ }^{9-10}$ se ha hecho hincapié en aspectos incluidos en las buenas prácticas de distribución que permiten prevenir la distribución inversa, tales como la correcta verificación de los proveedores y clientes por parte de todos los agentes de la cadena de distribución. Así mismo se incluyó en el Real Decreto 782/2013, de 11 de octubre, sobre distribución de medicamentos de uso humano, además de la obligación del cumplimiento de las mencionadas buenas prácticas, aspectos sobre la seguridad del transporte y las entregas en oficinas y servicios de farmacia así como la notificación de sospechas de desvíos de medicamentos. Requisitos dirigidos a hacer más difíciles estas prácticas de distribución inversa.

- Actuaciones contra estas prácticas: la identificación de las farmacias que desarrollaban estas actividades no ha sido sencilla dado que se han utilizado medios sofisticados para impedir la trazabilidad de las operaciones ilegales. Para contribuir a las actuaciones de las comunidades autónomas, la AEMPS les ha trasladado información sobre medicamentos con problemas de suministro debidos a la distribución, que ha servido en muchos casos para identificar establecimientos que desarrollaban estas prácticas. Por parte de las comunidades autónomas se han realizado cientos de inspecciones y solo en los últimos 3 años se han abierto 248 expedientes sancionadores.

Dada la complejidad de las inspecciones en establecimientos que disponían de sofisticados medios para evitar el acceso de los inspectores a la documentación que les implicaba en la actividad ilegal, ha sido crucial la intervención de las Fuerzas y Cuerpos de Seguridad del Estado, con los que la AEMPS, mantiene un convenio de colaboración en materia de tráfico ilícito de medicamentos desde el año 2010. Las complejas investigaciones y actuaciones de Guardia Civil y Policía Nacional han resultado decisivas para el desmantelamiento de diversas redes en los últimos años, de las que se han hecho eco los medios de comunicación. 
Es preciso mencionar que tanto la Federación Nacional de Asociaciones de Mayoristas y Distribuidores de Especialidades Farmacéuticas (FEDIFAR), como el Consejo General de Colegios Farmacéuticos han apoyado públicamente las actuaciones realizadas frente a estas redes con el fin de erradicar estas prácticas de distribución inversa.

- Colaboración e intercambio de información: el Comité Técnico de Inspección, adscrito a la AEMPS, ha desarrollado también un papel muy activo frente a estas prácticas. Desde 2008 se ha tratado este tema en 28 reuniones del comité y se han adoptado distintos documentos relativos a la coordinación e intercambio de información, siendo especialmente relevante y útil el continuo intercambio de experiencias, dado que estas redes operan con similares esquemas en todas las comunidades autónomas.

También ha sido muy importante la colaboración de las agencias de medicamentos de otros países de la Unión Europea, destinatarias de los medicamentos desviados y reintroducidos en el canal legal. Estas agencias han proporcionando a la AEMPS la información relativa a las notificaciones que reciben sobre el comercio paralelo y contribuido con ello a desenmascarar estas prácticas.

Para terminar quiero agradecer el gran esfuerzo de todos los inspectores que en circunstancias, en ocasiones, muy duras han trabajado para perseguir estas prácticas, y muy especialmente a las Fuerzas y Cuerpos de Seguridad del Estado, sin cuyas actuaciones no hubiera sido posible desmontar estas redes que, como se ha comentado, por su organización, al modo de bandas criminales, hacían imposibles los avances de las inspecciones en el ámbito administrativo.

Creo sinceramente que este desafío ha unido a todas las autoridades implicadas y hemos podido trabajar mejor por hacerlo juntas y así lo seguiremos haciendo hasta que la distribución inversa y sus riesgos para los pacientes sean un hecho del pasado.

\section{BIBLIOGRAFÍA}

1. Comisión Europea. Disponible en: http://ec.europa.eu/ health/human-use/50years/index_en.htm

2. Diario Oficial de la Unión Europea. Directiva 2011/62/ UE, del Parlamento Europeo y del Consejo, de 8 de junio de 2011, que modifica la Directiva 2001/83/CE por la que se establece un código comunitario sobre medicamentos de uso humano, en lo relativo a la prevención de la entrada de medicamentos falsificados en la cadena de suministro legal. DOUE num 174 de 1-07-2011.

3. Vazquez Mourelle R, Rodríguez Costa E, Pose Reino JM, Cadórniga Valiño L Tráfico inverso de medicamentos: experiencia en Galicia. Rev Esp Salud Pública 2015 89, 431-445.

4. Sentencia del Tribunal de Justicia (Sala Segunda) de 28 de junio de 2012, asunto C-7/11. Disponible en http://curia. europa.eu/juris/liste.jsf?td $=A L L \&$ language $=$ es $\&$ jur $=C, T, F$ \&num $=\mathrm{C}-7 / 11$

5. Diario Oficial de las Comunidades Europeas. Tratado de Funcionamiento de la Unión Europea. DOUE num 83 de 30-03-2010.

6. Agencia Española de Medicamentos y Productos Sanitarios. Circular $n^{0}$ 2/2012, Notificación previa de envíos de medicamentos a otros Estados miembros. Disponible en:http://www.aemps.gob.es/informa/circulares/ industria/2012/docs/circular_2-2012.pdf

7. Boletín Oficial del Estado. Real Decreto Legislativo $1 / 2015$, de 24 de julio, por el que se aprueba el texto refundido de la Ley de garantías y uso racional de los medicamentos y productos sanitarios. BOE num 177, de 25-7-2015.

8. Boletín Oficial del Estado. Ley Orgánica 1/2015, de 30 de marzo, por la que se modifica la Ley Orgánica 10/1995, de 23 de noviembre, del Código Penal. BOE num 77, de 31-3-2015.

9. Boletín Oficial del Estado. Ley 10/2013, de 24 de julio, por la que se incorporan al ordenamiento jurídico español las Directivas 2010/84/UE del Parlamento Europeo y del Consejo, de 15 de diciembre de 2010, sobre farmacovigilancia, y 2011/62/UE del Parlamento Europeo y del Consejo, de 8 de junio de 2011, sobre prevención de la entrada de medicamentos falsificados en la cadena de suministro legal, y se modifica la Ley 29/2006, de 26 de julio, de garantías y uso racional de los medicamentos y productos sanitarios. BOE núm 177 de 25-07-2013.

10. Boletín Oficial del Estado. Real Decreto 782/2013, de 11 de octubre, sobre distribución de medicamentos de uso humano. BOE num 251 de 19-10-2013. 